osteoclast (OC) differentiation but also to inhibit osteoblast differentiation.

Objectives Here we investigate the impact of activin a on joint destruction in rheumatoid arthritis.

Methods Synovial tissue samples from rheumatoid arthritis (RA) and osteoarthritis (OA) patients were analysed by immunohistochemical staining. For in vitro experiments, bone marrow-derived macrophages (BMM) were isolated from femurs and tibias of WT mice and differentiated into osteoclasts in the presence of macrophage colony-stimulating factor (M-CSF) and RANKL with or without activin A. OC differentiation was characterised by TRAP staining. Resorption activity was determined by quantification of osteoclast-mediated pit formation on a calcium phosphate-coated plate. Proliferation of BMMs was evaluated using the CyQUANT Cell Proliferation Assay Kit. Furthermore, osteoclast-specific gene expression as well as the activation of SMAD2, MAPK and NF-kB signalling were analysed by immunoblotting. The interaction of phospho-SMAD2 with NFATc1 was evaluated by co-immunoprecipitation using Dynabeads.

Results We demonstrate that activin A is highly abundant in the synovium of RA but not of OA patients. In vitro, activin A strongly enhanced the RANKL-mediated differentiation of BMMs into mature OCs, reflected by a significantly increased OC number, OC size and number of nuclei per OC compared to the conventional treatment with RANKL alone. Moreover, concomitant administration of activin A led to a significant increase of the total resorption area as well as resorption area per pit, indicating an increased activity of individual OCs. Effects of activin A on BMMs were not caused by increased proliferation since no effect on M-CSF-driven proliferation was observed. Furthermore, activin A alone was not able to induce the expression of OC differentiation markers, but the RANKL-induced expression was enhanced by activin A. After stimulation with activin A, BMMs showed an activation of SMAD2, but not of MAPK p38, ERK, JNK or NF-kB. Finally, co-stimulation of RANKL and activin A resulted in an increased interaction of activated SMAD2 with NFATc1.

Conclusions The data strongly suggest that increased expression of activin $\mathrm{A}$ in the arthritic joint is most likely associated with enhanced osteoclast formation, promoting joint destruction in rheumatoid arthritis.

Disclosure of interest None declared

\section{P074 IL-10 REGULATES SKIN THICKNESS AND SCALING IN IMIQUIMOD-INDUCED PSORIASIS-LIKE SKIN INFLAMMATION IN MICE}

${ }^{1} \mathrm{X} X \mathrm{U}^{*},{ }^{1} \mathrm{E}$ Prens, ${ }^{1} \mathrm{E}$ Florencia, ${ }^{2} \mathrm{~L}$ Boon, ${ }^{1} \mathrm{P}$ Asmawidjaja, ${ }^{1} \mathrm{~A}-\mathrm{M}$ Otten-Mus, ${ }^{1} \mathrm{E}$ Lubberts. ${ }^{1}$ Erasmus MC Rotterdam, Rotterdam; ${ }^{2}$ Bioceros, Utrecht, Netherlands

\subsection{6/annrheumdis-2018-EWRR2018.91}

Introduction Psoriasis is an autoimmune skin disease affecting around $0.6 \%$ to $3 \%$ of the whole population with detrimental physical and societal impacts. Previously, we established a psoriasis-like skin inflammation model in mice using topical application of imiquimod (IMQ). This model successfully recaptures all critical features of clinical psoriasis such as keratinocyte hyperproliferation, munro's microabscesses, and shares a similar infiltration profile of various immune cells. Previous data suggest up-regulation of IL-10, but its role in this psoriasiform model is not clear.
Objectives To investigate the role of IL-10 in the IMQinduced psoriasis-like skin inflammation.

Methods Psoriasis-like skin inflammation was induced by topical application of imiquimod (Aldara) for 5 or 10 days. Mice were injected intraperitoneally with anti-IL-10 or an isotype control antibody or subcutaneously with dexamethasone. Back skin of mice was scored for up to 10 days using a modified Psoriasis Area and Severity Index (PASI) score system adapted from clinical PASI score. Inflammation and skin thickness were scored histologically. Gene expression and immune cells in the skin were analysed using RT-PCR and flow cytometry, respectively.

Results At day 10, both skin thickness and scaling score were significantly higher after neutralising IL-10 compared to isotype control, or either group compared to dexamethasonetreated animals. At days 5 and $10, \mathrm{H}$ and $\mathrm{E}$ staining confirmed that epidermal thickness was more prominent in antiIL-10 treated mice compared to isotype control or dexamethasone-treated mice, with more profound differences at day 10 . Ki-67 staining for proliferating keratinocytes showed more proliferation at the epidermal basal layer after neutralising IL10. In addition, significant more infiltration of neutrophils was found in skin at day 10. At day 5, IL-23/IL-17 pathway cytokines were more significantly upregulated in anti-IL-10 group than the isotype control group, while at day 10, a significant upregulation of IL-19, IL-24 expression were found in anti-IL-10 group compared to isotype control.

Conclusions IL-10 regulates skin thickness and scaling during psoriasis-like skin inflammation. Furthermore, our data suggested that IL-10 might influence psoriatic symptoms through dampening of IL-23/IL-17 axis in early phase (day 5) and reducing IL-19 and IL-24 expression at late stage (day 10). The negative feedback signal of IL-10 partially explains the observed decrease of inflammation in imiquimod-induced skin inflammation after day 5 .

Disclosure of interest X. Xu: None declared, E. Prens: None declared, E. Florencia: None declared, L. Boon: None declared, P. Asmawidjaja: None declared, A.-M. Otten-Mus: None declared, E. Lubberts Grant/research support from: Novartis

\section{P075 CHANGES OF METABOLIC BIOMARKER LEVELS UPON ANTI-TNF THERAPY IN RHEUMATOID ARTHRITIS}

${ }^{1}$ A Pusztai ${ }^{*},{ }^{1} E$ Végh, ${ }^{1} A$ Horváth, 'S Szántó, 'G Szucs, ${ }^{1}$ A Hamar, ${ }^{1}$ A Domján, ${ }^{1} K$ Hodosi, ${ }^{2}$ Seres, ${ }^{3} \mathrm{G}$ Kerekes, ${ }^{1} \mathrm{Z}$ Szekanecz. ${ }^{1}$ Division of Rheumatology, Department of Medicine; ${ }^{2}$ Division of Metabolic Disease, Department of Medicine; ${ }^{3}$ Division of Intensive Care Unit, Department of Medicine, University of Debrecen, Faculty of Medicine, Debrecen, Hungary

\subsection{6/annrheumdis-2018-EWRR2018.92}

Introduction Rheumatoid arthritis (RA) has been associated with cardiovascular disease and metabolic syndrome. Numerous pro-inflammatory cytokines (e.g. TNF- $\alpha$, IL-1, IL-6) are released, which cytokines cause increased reactive oxygen species (ROS) production and thereby contribute to the increased lipid peroxidation and reduction of many antioxidants. These processes not only lead to the deterioration of joints and other tissues but may also contribute to comorbidities, such as atherosclerosis.

Objectives The aim of this study was to assess the effects of anti-TNF therapy on different metabolic markers, such as PON1 (paraoxonase 1), arylesterase, chemerin and 